\title{
Crystallization of a polymer on a surface
}

\author{
Jonathan P. K. Doye ${ }^{\mathrm{a})}$ and Daan Frenkel \\ FOM Institute for Atomic and Molecular Physics, Kruislaan 407, 1098 SJ Amsterdam, The Netherlands
}

(Received 6 May 1998; accepted 3 September 1998)

\begin{abstract}
We have studied the structure and free energy landscape of a semiflexible lattice polymer in the presence of the surface of a polymer crystal. At low temperatures coexistence of two-dimensional integer-folded crystals is observed. As the temperature is increased there is a transition from these crystalline configurations to a disordered coil adsorbed onto the surface. The polymer then gradually develops a three-dimensional character at higher temperatures. We compute the free energy as a function of increasing crystallinity and compare with the free energy profiles assumed by the Lauritizen-Hoffman surface nucleation theory of polymer crystallization. Our free energy profiles exhibit a "sawtooth" structure associated with the successive formation of chain folds. However, in the early stages of crystallization our profiles significantly deviate from those assumed by surface nucleation theory because the initial nucleus is not a single stem but two incomplete stems connected by a fold. This finding has significant implications for the theoretical description of polymer crystallization. (C) 1998 American Institute of Physics. [S0021-9606(98)52546-9]
\end{abstract}

\section{INTRODUCTION}

On crystallization from solution or the melt simple polymers typically form lamellar crystals. As the backbone of the polymer chain is oriented perpendicular to the plane of the lamellar, and yet the thickness of the crystals is smaller than the length of the chain, a single polymer must traverse the crystal many times folding back on itself at each surface. ${ }^{1}$ Furthermore the thickness of the crystals have a wellcharacterized dependence on the degree of supercooling. The thickness is always slightly larger than the minimum thickness for which a lamellar crystal is thermodynamically stable.

However, although these simple facts were discovered about 40 years ago, there is still no consensus on their theoretical explanation. ${ }^{2}$ Moreover, two of the more dominant approaches-Lauritzen-Hoffman surface nucleation theory ${ }^{3-5}$ and the entropic barrier model ${ }^{6-9}$-appear irreconcilable. One of the difficulties is that the theories have to make specific assumptions about the microscopic mechanisms of crystallization. As it is difficult to probe these processes directly in an experiment, the main test for the theories has been the comparison of their predictions with macroscopic properties, such as the crystal thickness, growth rate, and shape. But these tests have not proved discriminating enough; the two main theories (perhaps with the help of various refinements or "suitable" choices of parameter) have both been able to provide an adequate description of many of the macroscopic properties.

Therefore, (atomistic) simulation can potentially play an important role in this debate by providing insight into the microscopic processes involved in polymer crystallization, and thus help in the critical assessment and refinement of the current theories and perhaps in the development of new theo-

\footnotetext{
${ }^{a}$ Present address: University Chemical Laboratory, Lensfield Road, Cambridge CB2 1EW, UK.
}

ries. However, there have been few simulation studies ${ }^{10-13}$ which have attempted to investigate the basic process in solution crystallization, the adsorption and crystallization of a polymer on the growth surface. (There are many studies which just consider adsorption. ${ }^{14-21}$ ) Although this is a nonequilibrium process, as a first step it is useful to understand the equilibrium behavior of a single polymer in the presence of the surface of a polymer crystal. In this paper, we first study the basic thermodynamic properties of a simple lattice model of such a system (Sec. III). This will also add to the increasing knowledge of the rich phase behavior of single homopolymer chains; in particular, we draw out the similarities and differences from the behavior of an isolated semiflexible polymer, a case that has received much more attention. ${ }^{22-29}$ Second, we examine in detail the free energy profile for the crystallization pathway suggested by surface nucleation theory (Sec. IV).

\section{METHODS}

\section{A. Polymer model}

In our model the polymer is represented by an $N$-unit self-avoiding walk on a simple cubic lattice. There is an attractive energy, $-\epsilon$, between nonbonded polymer units on adjacent lattice sites and between polymer units and the surface, and an energetic penalty, $\epsilon_{g}$, for kinks in the chain. The total energy is given by

$$
E=-\left(n_{\mathrm{pp}}+n_{\mathrm{ps}}\right) \epsilon+n_{g} \epsilon_{g},
$$

where $n_{\mathrm{pp}}$ is the number of polymer-polymer contacts, $n_{\mathrm{ps}}$ is the number of polymer-surface contacts and $n_{g}$ is the number of kinks or "gauche bonds" in the chain. We choose the energy for polymer-polymer and polymer-surface contacts to be the same so that the surface represents the surface of a polymer crystal. $\epsilon$ can be considered to be an effective interaction representing the combined effects of polymerpolymer, polymer-solvent, and solvent-solvent interactions, 
and so our model is a simplified representation of a semiflexible polymer at the interface between a polymer crystal and solution. The behavior of the polymer is controlled by the ratio $k T / \epsilon$; large values can be considered as either high temperature or good solvent conditions, and low values as low temperature or bad solvent conditions. The parameter $\epsilon_{g}$ defines the stiffness of the chain. The polymer chain is fully flexible at $\epsilon_{g}=0$ and becomes stiffer as $\epsilon_{g}$ increases. In this study we only consider $\epsilon_{g} \geqslant 0$.

This polymer model has been recently used to study the effects of stiffness on the phase behavior of isolated homopolymers by theory ${ }^{25}$ and simulation, ${ }^{26,28}$ and also in kinetic Monte Carlo simulations of the growth of polymer crystals. ${ }^{30,31}$

The global potential energy minimum at a particular (positive) $\epsilon_{g}$ is determined by a balance between maximizing $n_{\mathrm{pp}}$ and $n_{\mathrm{ps}}$, and minimizing $n_{g}$; it is a folded structure that lies flat on the surface. If the polymer is able to form a structure that is a two-dimensional rectangle with dimensions $a \times b(N=a b)$, where $a \leqslant b$, then

$$
\begin{aligned}
& n_{\mathrm{ps}}=N, \\
& n_{\mathrm{pp}}=N-a-b+1, \\
& n_{g}^{\min }=2 b-2 .
\end{aligned}
$$

The structures that correspond to $n_{g}=n_{g}^{\min }$ have the polymer chain folded back and forth along the longer dimension of the rectangle. By minimizing the resulting expression for the energy one finds that the lowest energy polymer configuration should have

$$
\frac{b}{a}=1+\frac{2 \epsilon_{g}}{\epsilon} .
$$

Therefore, at $\epsilon_{g}=0$ the ideal shape is a square and for positive $\epsilon_{g}$ a rectangle extended in one direction, the aspect ratio of which increases as the chain becomes stiffer. However, at most sizes and values of $\epsilon_{g}$ it is not possible to form a rectangle with the optimal aspect ratio. Nevertheless, it is easy to find the global minimum just by considering the structures which most closely approximate this optimal shape.

\section{B. Simulation techniques}

To simulate our system we use a configurational-bias Monte Carlo ${ }^{32}$ technique including moves in which a midsection of the chain is regrown. ${ }^{33} \mathrm{We}$ also make occasional bond-flipping moves which, although they do not change the shape of the volume occupied by the polymer, change the path of the polymer through that volume. ${ }^{28,34,35}$ These moves speed up equilibration in the dense phases. During the simulation we always constrain the polymer to have at least one unit in contact with the surface. This constraint prevents the polymer from becoming detached from the surface at the higher temperatures used in Sec. III; it has no effect at the temperatures used in Sec. IV. Thermodynamic properties, such as the heat capacity, were calculated from the energy distributions of each run using the multihistogram method. ${ }^{36,37}$
In order to monitor the state of the polymer we devised two order parameters. The first, $Q_{2 \mathrm{D}}$, probes the orientational order within the two-dimensional polymer adsorbed onto the surface,

$$
Q_{2 D}=\sqrt{2 \sum_{\alpha=x, y}\left(\frac{n_{\alpha}}{n_{x}+n_{y}}-\frac{1}{2}\right)^{2}},
$$

where $x$ and $y$ are in the plane of the surface and $n_{\alpha}$ is the number of bonds in the direction $\alpha . Q_{2 \mathrm{D}}$ has a value of 1 if all the bonds are in the same direction, i.e., the polymer has a linear configuration, and a value of 0 if the bonds are oriented isotropically in the plane. The second order parameter, $Q_{3 \mathrm{D}}$, probes the dimensionality of the polymer.

$$
Q_{3 \mathrm{D}}=\sqrt{6\left[2\left(\frac{n_{x}+n_{y}}{2(N-1)}-\frac{1}{3}\right)^{2}+\left(\frac{n_{z}}{(N-1)}-\frac{1}{3}\right)^{2}\right]} .
$$

$Q_{3 \text { D }}$ has a value of 1 if all the polymer units are in contact with the surface and a value of 0 if the bonds are oriented isotropically in space.

In Sec. IV we compute the free energy along pathways characterized by an order parameter, $N_{x \text { tal }}$, which measures the degree of crystallinity. This Landau free energy is simply related to the canonical probability distribution for the order parameter:

$$
A_{L}\left(N_{x \text { tal }}\right)=A-k T \log p_{\text {can }}\left(N_{x t \text { tal }}\right),
$$

where $A$ is the Helmholz free energy. However, all relevant regions of this distribution are not significantly sampled in the canonical ensemble, and so we use umbrella sampling ${ }^{38}$ to calculate the free energy accurately over the whole range of the order parameter. This is achieved by multiplying the Boltzmann factor by the exponential of a biasing distribution, $W\left(N_{x \text { tal }}\right)$, i.e., the simulation samples configurations with a probability proportional to $\exp \left(-\beta E+W\left(N_{x t a l}\right)\right)$. The canonical probability distribution is then obtained from the probability distribution from the biased run, $p_{\text {multi }}\left(N_{x \text { tal }}\right)$, by

$$
p_{\text {can }}\left(N_{x \text { tal }}\right)=p_{\text {multi }}\left(N_{x \text { tal }}\right) \exp \left(-W\left(N_{x \text { tal }}\right)\right) \text {. }
$$

We wish to choose $W$ such that $p_{\text {multi }}\left(N_{x \text { tal }}\right)$ is approximately constant over the whole range of $N_{x t a l}$ (the so-called multicanonical approach $\left.{ }^{39,40}\right)$. However, this only occurs when $W\left(N_{x \text { tal }}\right) \approx A_{L}\left(N_{x \text { tal }}\right) / k T$ and so we have to construct $W$ iteratively from the results of a number of short preliminary simulations. $^{41}$

\section{THERMODYNAMICS}

In the presentation of our results we concentrate on one example, a 200-unit polymer with $\epsilon_{g}=4 \epsilon$. Results for other positive values of $\epsilon_{g}$ show the same basic behavior. We also mainly dwell on those aspects of the thermodynamics which are relevant to polymer crystallization or differ markedly from the behavior of the isolated homopolymer. ${ }^{25,26,28}$

At zero temperature the global potential energy minimum has the lowest free energy. For $\epsilon_{g}=4 \epsilon$ the optimal aspect ratio of a crystal is 9 [Eq. (3)]. For $N=200$ the crystal that most closely approximates this shape has five stems (a stem is a straight section of the polymer) which are 40 units long; it is the global minimum. The example shown in Fig. 


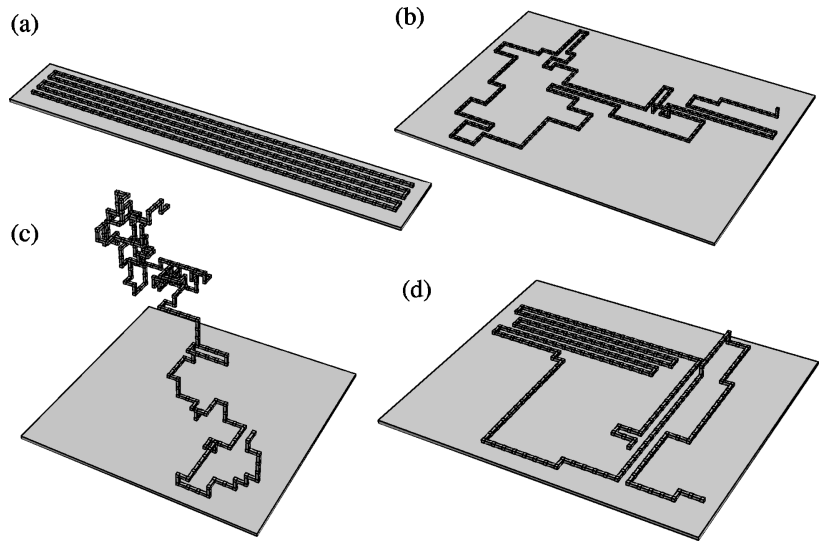

FIG. 1. Some representative configurations for a 200-unit polymer in the presence of a surface. (a) Crystalline configuration with five stems of length 40 units; it is one of the lowest energy configurations for $\epsilon_{g}=4 \epsilon \cdot Q_{2 \mathrm{D}}$ $=0.960$ and $R_{g}^{2}=135.3$. (b) Disordered two-dimensional coil on the surface produced in a run at $T=2.5 \epsilon k^{-1} \cdot Q_{2 \mathrm{D}}=0.235$ and $R_{g}^{2}=141.6$. (c) Threedimensional coil produced in a run at $T=5.0 \epsilon k^{-1} . Q_{3 \mathrm{D}}=0.010$ and $R_{g}^{2}$ $=99.1$. (d) Configuration from a simulation at $T=2.375 \epsilon k^{-1}$ in which half the configuration is crystalline and half is disordered.

1(a) has a tight fold at the end of each stem, a situation which is often referred to as adjacent reentry, but there are a number of degenerate configurations with nonadjacent reentry. The differences in energy between the various crystalline configurations are only small and so at low temperatures more than one type of crystalline configuration is observed in the simulation; they can be differentiated by their radius of gyration. Figure 2(b) shows the presence of four coexisting crystallites which have four, five, six, or seven complete stems. This preference for crystalline configuration with complete stems is energetic in origin-it maximizes the number of polymer-polymer contacts for each fold. A similar trend is observed in long monodisperse alkanes; the crystals have preferred thicknesses which correspond to an integer number of complete stems. ${ }^{42,43}$ This effect has also been observed in simulations of these systems. ${ }^{11,13}$

As the temperature is increased configurations with more disorder in the stem length and a shorter average stem length are entropically more favored. This causes the peaks in the probability distribution of the radius of gyration to broaden and the radius of gyration to decrease in the range $T$ $=1.0-1.75 \epsilon k^{-1}$ [Fig. 2(a)].

As with the isolated homopolymers there must come a point when disordered configurations become more favored than crystalline configurations. This "melting" transition is signaled by a heat capacity peak (Fig. 3) and by a loss of orientational order [Fig. 4(a)]. Although $Q_{2 \mathrm{D}}$ drops down to a value of 0.1 at the transition, $Q_{3 \mathrm{D}}$ remains close to one. This shows that on melting the polymer adopts a twodimensional configuration on the surface with no orientational order in the plane [Fig. 1(b)]. Furthermore, although this order-disorder transition is a finite size analog of a bulk first-order phase transition, no bimodality is seen in the probability distribution of $Q_{2 \mathrm{D}}$ [Fig. 4(b)], i.e., there is no free energy barrier between states with high and low $Q_{2 \mathrm{D}}$. Near to the transition temperature, $T_{m}$, the $Q_{2 \mathrm{D}}$ probability distribution is very broad and flat, perhaps because of the contri- (a)
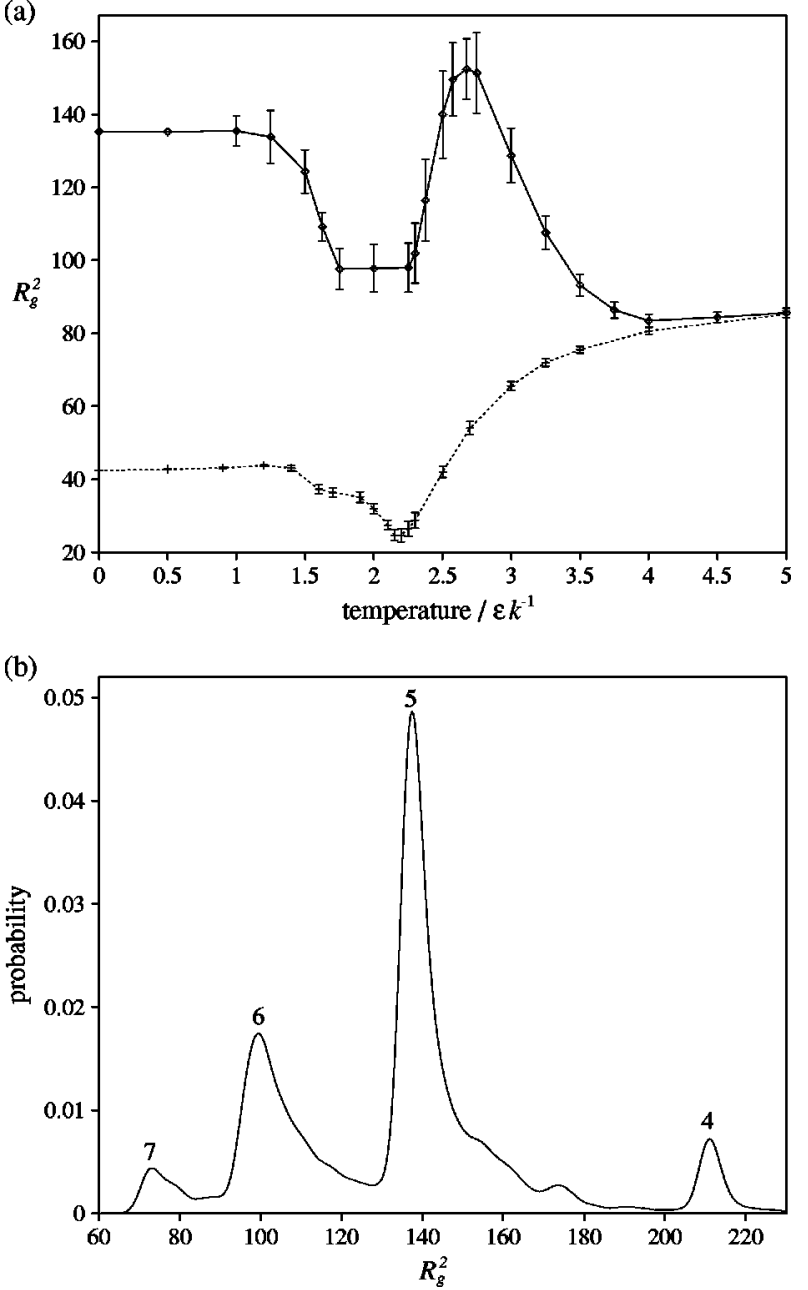

FIG. 2. (a) $R_{g}^{2}$ as a function of temperature for a 200-unit polymer at $\epsilon_{g}$ $=4 \epsilon$. For comparison the results for an isolated polymer (dashed line) have also been included. (b) Probability distribution for $R_{g}^{2}$ at $T=1.25 \epsilon k^{-1}$. The peaks are labeled by the numbers of stems in the corresponding configurations.

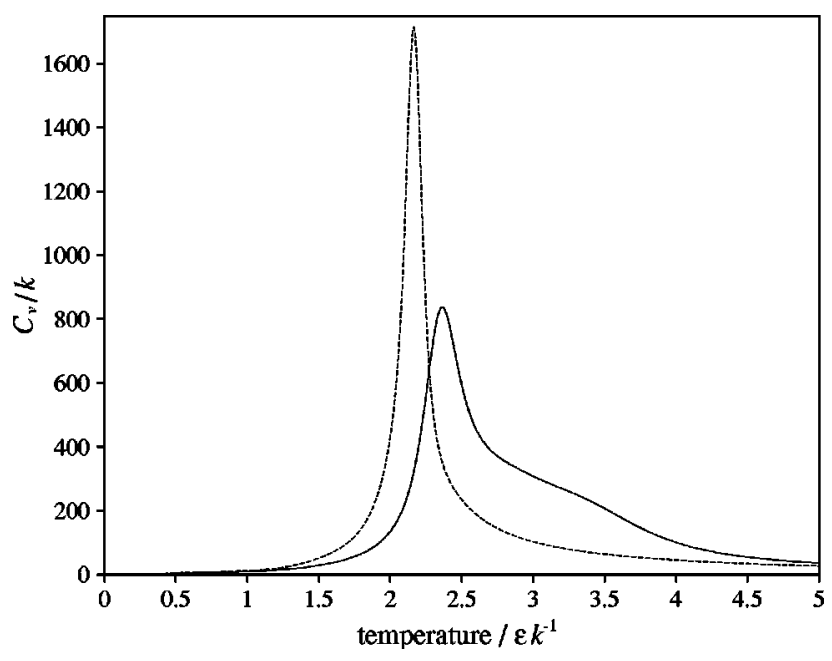

FIG. 3. $C_{v}$ as a function of temperature for a 200-unit polymer at $\epsilon_{g}$ $=4 \epsilon$. For comparison the results for an isolated polymer (dashed line) have also been included. 


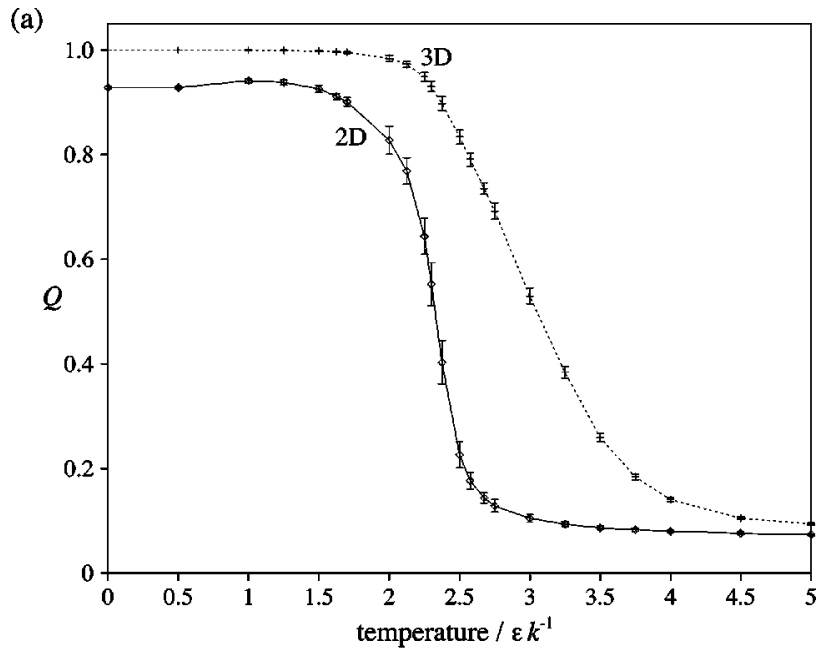

(b)

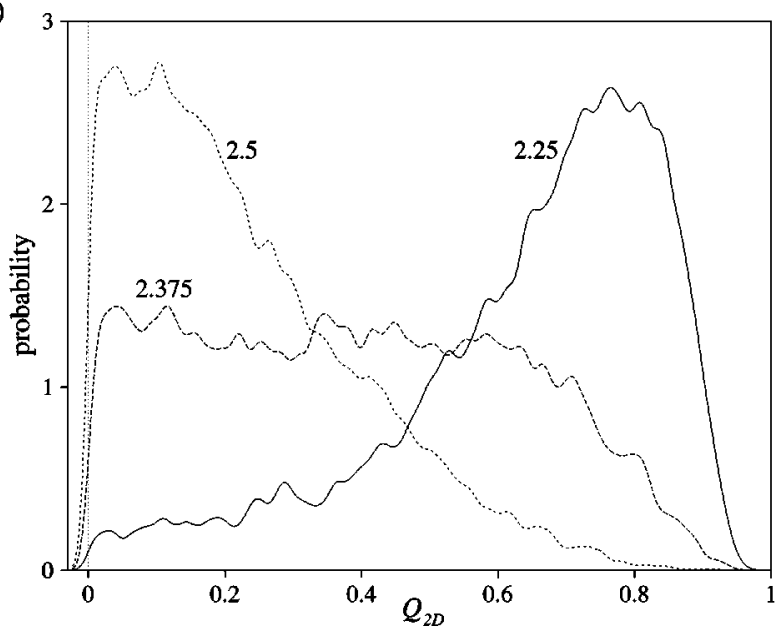

FIG. 4. (a) $Q_{2 \mathrm{D}}$ and $Q_{3 \mathrm{D}}$ as a function of temperature for a 200-unit polymer at $\epsilon_{g}=4 \epsilon$. (b) Probability distributions for $Q_{2 \mathrm{D}}$ at three different temperatures around the melting temperature, as labeled.

bution of partly crystalline, partly disordered configurations [such as the one depicted in Fig. 1(d)] with intermediate values of $Q_{2 \mathrm{D}}$.

The order-disorder transition also causes the radius of gyration to increase in the range $T=2.3-2.7 \epsilon k^{-1}$, first as the polymer passes to the disordered state and then as this coil begins to expand with temperature. However, this rise is checked when the polymer begins to develop a threedimensional character [Fig. 1(c)]. The change in dimensionality occurs gradually and is signaled by a decrease in $Q_{3 \mathrm{D}}$ and also a decrease in the radius of gyration to a value which is comparable to that for the an isolated chain of the same size [Fig. 2(a)]. The associated loss in polymer-surface contacts is the cause of the high temperature shoulder in the heat capacity. Such desorption transitions are well-understood ${ }^{14}$ and we do not dwell on its features here.

In Figs. 2 and 3 we have included results for an isolated polymer for comparison. Until the transition to a threedimensional configuration, the $R_{g}^{2}$ curves for the two cases have a similar form [except that the values are larger for the two-dimensional (2D) polymer on the surface] due to the similar nature of the transitions in the two cases. ${ }^{30}$ The heat capacity peak for the isolated polymer is sharper, suggesting

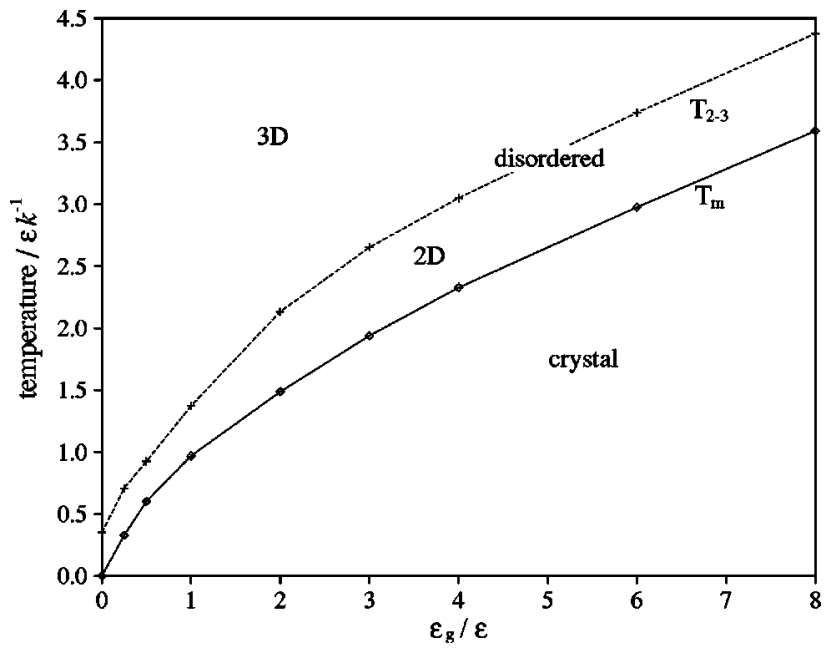

FIG. 5. Phase diagram of a 200-unit polymer on a surface. The phase diagram is divided into regions by the values of the melting point, $T_{m}$, and by the transition from two- to three-dimensional configurations, $T_{2-3}$. These two transition temperatures are defined by $Q_{2 \mathrm{D}}\left(T_{m}\right)=0.5$ and $Q_{3 \mathrm{D}}\left(T_{2-3}\right)$ $=0.5$. No attempt has been made to differentiate between coils and globules in the disordered region of the phase diagram.

that the melting transition has a stronger first-order-like character. Similarly, it is easier to find an order parameter for the isolated polymer that shows bimodality (i.e., the presence of a free energy barrier) in the transition region. This difference is probably because the energy difference between ordered and disordered states is less in the presence of the surface; the energy of the two-dimensional coil is reduced relative to the three-dimensional coil due to the interaction energy with the surface.

The basic behavior described above generally holds for any positive $\epsilon_{g}$, as be can seen from the phase diagram in Fig. 5. In particular the order-disorder transition always leads to a two-dimensional disordered polymer adsorbed onto the surface, which only at higher temperature becomes three dimensional. This fact could be of particular relevance to polymer crystallization from solution. It suggests that a polymer forms a two-dimensional configuration on the growth surface before crystallizing, rather than crystallizing directly from solution. The thermodynamics of the crystallization process will depend significantly on which of these two possible mechanisms holds. The direct mechanism is implicitly or explicitly assumed in several theoretical descriptions. ${ }^{2}$ Yet, the present results appear to support the assumption made by Yamamoto in his study of the dynamics of the crystallization process. ${ }^{10}$

However, it is not clear whether this aspect of our results - crystallization being preceded by adsorption-is likely to be representative of the behavior of real polymers; it might reflect some of the simplicities in the model. For example, in our model an adsorbed disordered polymer and a crystallite can have the same energy of interaction with the surface, whereas in reality a polymer chain that fits snugly into a groove formed by chains in the crystalline surface will have a lower energy than a random configuration on the surface. Such features could result in crystallization and adsorption occurring simultaneously. Therefore, it would be useful 
if more realistic simulations were performed to clarify this situation.

In the phase diagram in Fig. 5 we have not attempted to distinguish regions where the scaling behavior of the disordered state is that of a coil or of a globule. The main differences in behavior between polymers with different values of $\epsilon_{g}$ are related to the position of the coil-globule transitions with respect to the adsorption transition and show up most clearly in quantities such as the radius of gyration. For example, the maximum in $R_{g}^{2}$ for the two-dimensional disordered polymer that occurs at $\epsilon_{g}=4 \epsilon$ (Fig. 2) does not occur for all $\epsilon_{g}$. However, we do not pursue these issues further here.

In this study the surface represents the surface of a polymer crystal. However, if different interaction energies were assigned to polymer-polymer and polymer-surface contacts this model could be used to examine the phase behavior of a polymer in the presence of a surface of a different material. In particular, changing the polymer-surface interaction is likely to effect the relative positions of the adsorption and crystallization transitions compared to the current model. ${ }^{44}$ For example, a decrease in the energy of polymer-surface contacts would destabilize the adsorbed phase and for a large enough decrease this would lead to crystallization occurring direct from a three-dimensional configuration in solution.

\section{FREE ENERGY FOR A SINGLE CRYSTALLIZATION PATHWAY}

Having understood the basic thermodynamics of our system we are now in a position to use the model to examine in detail the free energy landscape for the microscopic mechanism of polymer crystallization assumed by the LauritzenHoffman surface nucleation theory. ${ }^{3-5}$ In this theory the key process in determining the thickness of the lamellar crystals is considered to be the nucleation and growth of a new polymer layer on the crystal growth face (the thin edges of the lamellae). In the theory the growth rate for new layers of different thicknesses are compared. It is argued that crystals with a thickness close to that which gives the maximum growth rate will dominate the ensemble of possible crystals, and so the predicted thickness of the crystal corresponds approximately to the thickness for which the rate is a maximum.

To calculate the growth rates some assumptions have to be made about the processes involved and the associated thermodynamics. First, the new layer is assumed to grow by the addition of a succession of stems along the growth face each connected to the previous by a tight fold. Second, the length of each stem is assumed to be the same as the thickness of the lamella. Third, to calculate the free energy for this process macroscopic thermodynamic properties are used. This allows the free energy as a function of the number of stems to be written as

$$
A_{L}\left(N_{\text {stem }}\right)=2 b l \sigma+2\left(N_{\text {stem }}-1\right) a b \sigma_{f}-a b l \Delta F,
$$

where $\sigma$ is the surface free energy of the growth surfaces, $\sigma_{f}$ is the free energy of the fold surfaces, $\Delta F$ is the free energy of crystallization per unit volume, $l$ is the thickness of the lamella, $a$ is the width of a stem, and $b$ is the depth of a stem.

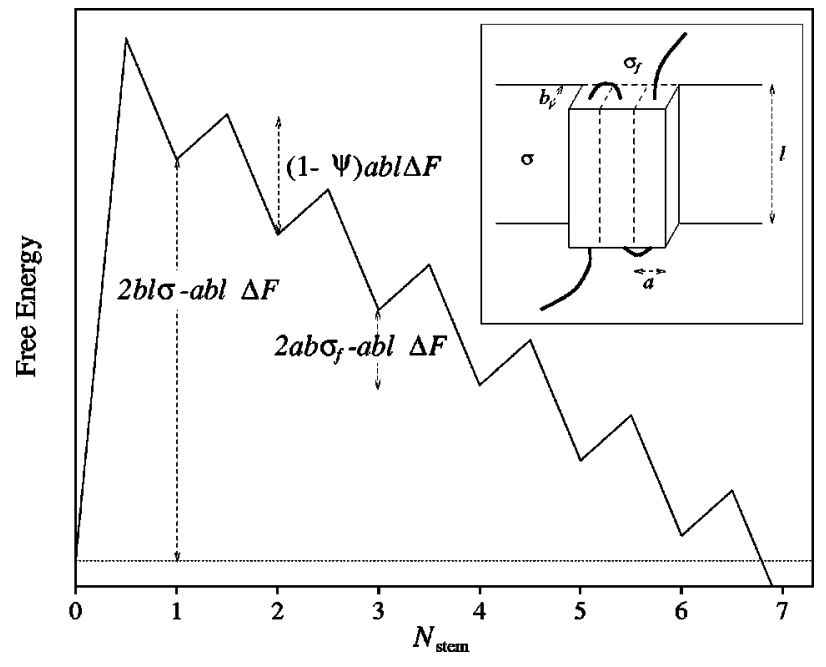

FIG. 6. Free energy profile assumed by the Lauritzen-Hoffman surface nucleation theory of polymer crystallization. The theory considers the crystal to form by laying down of adjacent stems of the polymer. The inset is a schematic representation of a configuration where three stems have been deposited. The profile is for a temperature at which the crystal is the most stable state.

The geometry of the assumed mechanism is depicted in the inset of Fig. 6. The first term in Eq. (8) corresponds to the creation of the two new lateral surfaces on either side of the nucleus. This free energy has to be "paid for" on the laying down of the first stem. The second term corresponds to the free energy of the folds that have to be created on the deposition of subsequent stems.

The fourth assumption is that at the barrier between states with different $N_{\text {stem }}$ all the new surfaces have been created and that a fraction $\Psi$ of the free energy of crystallization has been released. These assumptions lead to free energy profiles like that depicted in Fig. 6.

There have been a number of specific criticisms of this free energy profile. First, the model assumes that the value of $\Psi$ for deposition of the first and subsequent stems is the same when clearly the physical processes for these two cases are very different. The main motivation for this assumption is simply that it allows an analytical solution to the theory. More recently, there has been some work which has attempted to find approximate solutions when this simplifying assumption is relaxed. ${ }^{45,46}$

Second, for any nonzero value of $\Psi$ the predicted thickness becomes infinite at sufficiently large supercoolings-the so-called $\delta l$ catastrophe. This occurs when there is no longer any barrier for the deposition of the first stem, i.e., for temperatures where $\Psi>2 \sigma /(a \Delta F)$. To avoid this unwanted behavior, the theory has been supplemented with a justification for a zero value of $\Psi$. The argument is that the chain first forms a weakly physisorbed aligned state which traverses the growth face (such a configuration has a high free energy because it has lost the entropy associated with orientational disorder) before it crystallizes to form the first crystalline stem, ${ }^{5}$ rather than the free energy of crystallization being released as successive units of the first stem are deposited onto the surface. There seems little evidence to favor the first scenario except that it avoids a $\delta l$ catastrophe appearing in 
the theory. Furthermore, the second mechanism would be expected to involve a lower free energy pathway, and so, if feasible, would be preferred.

If simulations are to help us make some critical assessment of the free energy profile used in the surface nucleation theory we need to devise an order parameter which can act as a reaction coordinate along a pathway to a specific crystal. We use $N_{x t a l}$, which we define as the number of units in the largest fragment of the polymer that has part of the structure of our target crystal. This target crystal has adjacent reentry of the stems which are all of a specified length, $l$. As our reaction coordinate is more fine-grained than that used in the surface nucleation theory it allows us to find the location of any free energy transition states on the path.

To compare with the theoretical free energy profile the pathway should go from the disordered state of the polymer to the target crystal. However, the difficulty with the order parameter, $N_{x \text { tal }}$, is that it is not able to distinguish between configurations which are disordered and those that have a crystalline structure different from the target crystal. Therefore, we have to introduce some constraints to ensure that the $N-N_{x \text { tal }}$ units which are not part of the largest fragment of the target crystal adopt a disordered configuration. We use the following order parameters to achieve this: $Q_{2 D}^{\text {rest }}, Q_{\|}^{\text {rest }}$, and $Q_{x r} . Q_{2 \mathrm{D}}^{\text {rest }}$ is simply the same as $Q_{2 \mathrm{D}}$ except that only bonds in the noncrystalline part of the polymer are taken into account. $Q_{\|}^{\text {rest }}=n_{\|}^{\text {rest }} / 2 N_{\text {rest }}$, where $n_{\|}^{\text {rest }}$ is the number of instances when a bond in the noncrystalline part of the polymer is adjacent and parallel to another bond on the surface, and $N_{\text {rest }}=N-N_{x \text { tal }} \cdot Q_{x r}=n_{x r} / N_{x \text { tal }}$, where $n_{x r}$ is the number of contacts between the crystalline part of the chain and the rest of the polymer.

The constraints we use are: $Q_{2 \mathrm{D}}^{\text {rest }}<0.3, Q_{\|}^{\text {rest }}<0.3$ and $Q_{x r}<0.15$. Low values of $Q_{2 \mathrm{D}}^{\text {rest }}$ and $Q_{\|}^{\text {rest }}$ are appropriate for a disordered chain. (The upper bounds have been found to be reasonable from monitoring these order parameters for the purely disordered state.) The constraint on $Q_{x r}$ prevents the nucleus from being stabilized by a more locally ordered region surrounding it. However, the exact values for these constraints are slightly arbitrary. The free energy profiles that we obtain do reflect these choices to a certain extent, but only in the numerical details and not in their basic structure.

In Fig. 7(a) we show some free energy profiles that we obtain for the formation of a target crystal with stems that are 40 units long at a variety of temperatures. It is immediately apparent that the profiles have a sawtooth structure similar to the surface nucleation profiles. The minima occur at $2 l, 3 l$, and $4 \mathrm{l}$. Presumably, for longer polymers this structure would be repeated at higher multiples of $l$. [Indeed when we use $l=25$ minima up to $7 l$ are observed (Fig. 7(b)]. After the minimum the free energy rises sharply with the incorporation of the next two polymer units into the crystal reaching a maximum at $n l+2$ or $n l+3$. These steps in the free energy have a clear interpretation. Configurations with a complete number of stems are favored because such a configuration maximizes the number of polymer-polymer contacts relative to the number of folds in the crystal. To increase the size of the crystal fragment a new fold has to be formed with an accompanying rise in the free energy. After this there is a
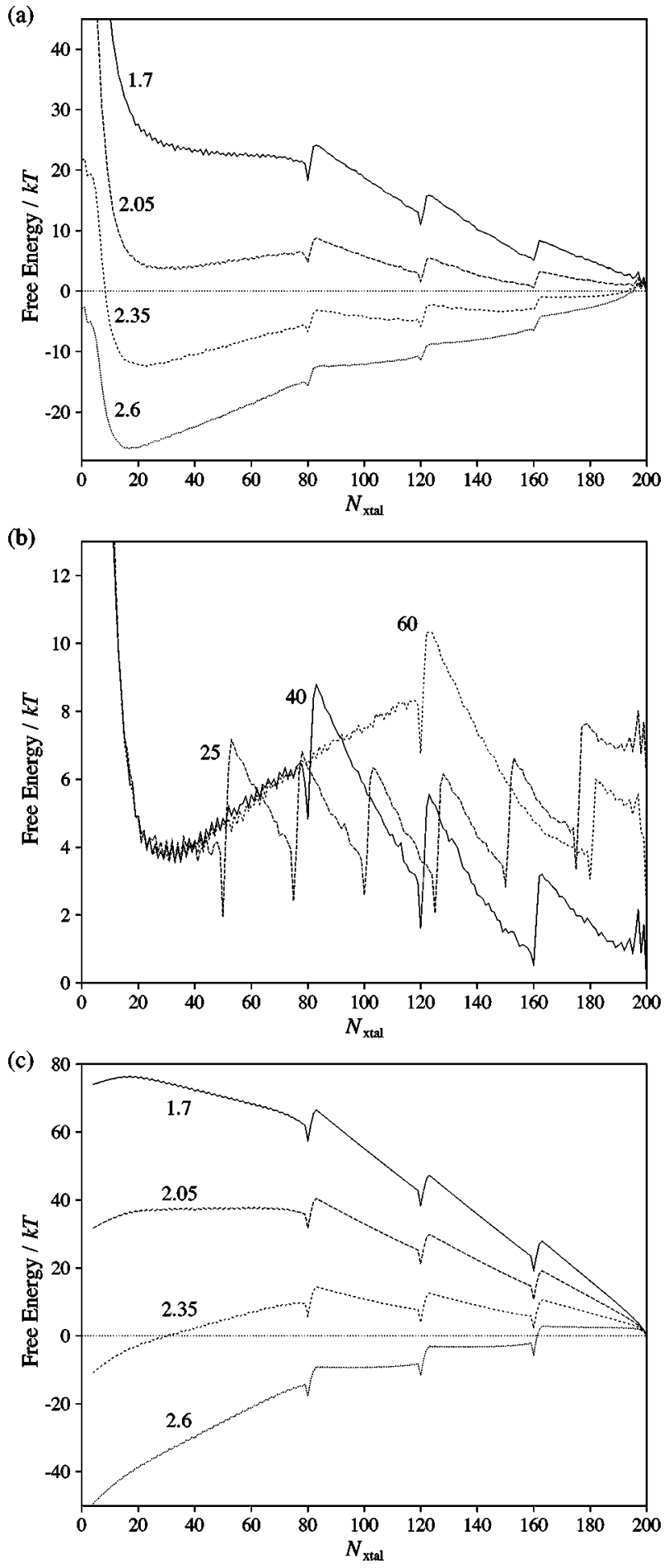

FIG. 7. Free energy for the formation of a target crystal with equal stem lengths and adjacent reentry. In (a) and (c) the target crystal has stems 40 units long; the four curves correspond to different temperature, as labeled, and we have set the zero of free energy to that for the complete crystal. In (b) the three curves correspond to target crystals with different stem length, as labeled, all at $T=2.05 \epsilon k^{-1}$; we have set the zero of free energy to that for the complete crystal with stem length 40 , and the free energy differences between the different complete crystals were calculated by complete enumeration of possible configurations. The free energy profiles in (a) and (b) are from simulation and those in (c) have been calculated using Eq. (9).

monotonic decrease in the free energy as the new stem grows until the next fold must form. Translating this into the language used in the surface nucleation approach, $\Psi\left(N_{\text {stem }}\right.$ $\left.\rightarrow N_{\text {stem }}+1\right) \approx 0$ for $N_{\text {stem }} \geqslant 2$ and the transition state occurs 
virtually immediately after the completion of the previous stem.

However, at $N_{x \text { tal }}<2 l$ there are major differences between the simulation and the surface nucleation free energy profiles. First, there is no sign in the simulation results of any feature at $N_{x \mathrm{tal}}=l$ (or at any other value of $N_{x \mathrm{tal}}$ ) due to the formation of the first fold. Therefore, the mechanism of its formation must be significantly different from that for subsequent folds; it does not occur abruptly at a specific value of $N_{x t a l}$. In the surface nucleation approach it is assumed that new stems are laid down one at a time. It does not allow for the possibility that two new stems could be formed simultaneously. There is no such restriction in our simulations. We find that the initial nucleus is not a single stem but two incomplete stems of approximately equal length connected by a fold. As $N_{x \text { tal }}$ increases the two stems grow in length simultaneously. The reason for this behavior is energetic. It is simple to show that a two-stem nucleus can have a lower energy than a single-stem nucleus when $N_{x \text { tal }}>4 \epsilon_{g} / \epsilon+2$. Confirmation of this behavior can be found from Fig. 8(c). The number of gauche bonds in the crystal has risen to two by $N_{x \text { tal }} \sim 25$. The possibility of such a two-stem nucleus has previously been suggested by Point. ${ }^{47}$

We should note that it is possible that the size of the surface may have a small effect on the competition between the two possible nuclei. On our "infinite", surface the position of the nucleus is irrelevant. However, on the growth surface of a lamellar crystal it is important that the fold of the two-stem nucleus is close to (or at) the edge of the lamella, whereas the initial position of a single-stem nucleus is unimportant. Having said this a similar preference for two-stem nuclei has been observed in kinetic Monte Carlo simulations of polymer crystallization on the growth surface of a lamellar crystal. $^{31}$

Second, the behavior at small $N_{x \text { tal }}$ is different. For example at $T=1.7 \epsilon k^{-1}$ the free energy is downhill until the barrier at $N_{x \mathrm{tal}}=2 l$. For the higher temperature free energy profiles there is a free energy minimum corresponding to the disordered state but it occurs at a finite value of $N_{x \mathrm{tal}}$, which decreases with increasing temperature. At $T=2.05,2.35$, and $2.6 \epsilon k^{-1}$ the minima are at $N_{x \text { tal }}=33,23$, and 17 , respectively. Furthermore the free energy rises very steeply as $N_{x \text { tal }}$ decreases toward zero. These effects occur because crystallization is occurring from a disordered state which is adsorbed onto the surface. This disordered state has structural motifs - single straight sections and short folds-which correspond to small fragments of the target crystal. For example the two-dimensional disordered conformation shown in Fig. 1(b) has $N_{x \mathrm{tal}}=26$. As the temperature decreases the persistence length of the polymer increases and so the value of $N_{x \text { tal }}$ associated with the coil increases.

When there is a free energy minimum corresponding to the coil, for values of $N_{x \text { tal }}$ beyond the minimum the free energy rises linearly until $N_{x \mathrm{tal}}=2 l$. Therefore, in this temperature range the barrier to forming a crystal nucleus with two complete stems does increase with $l$, as is clearly shown in Fig. 7(b). However, this is very different from the $l$ dependence of the initial free energy barrier that is predicted by surface nucleation theory; there the top of the initial surface
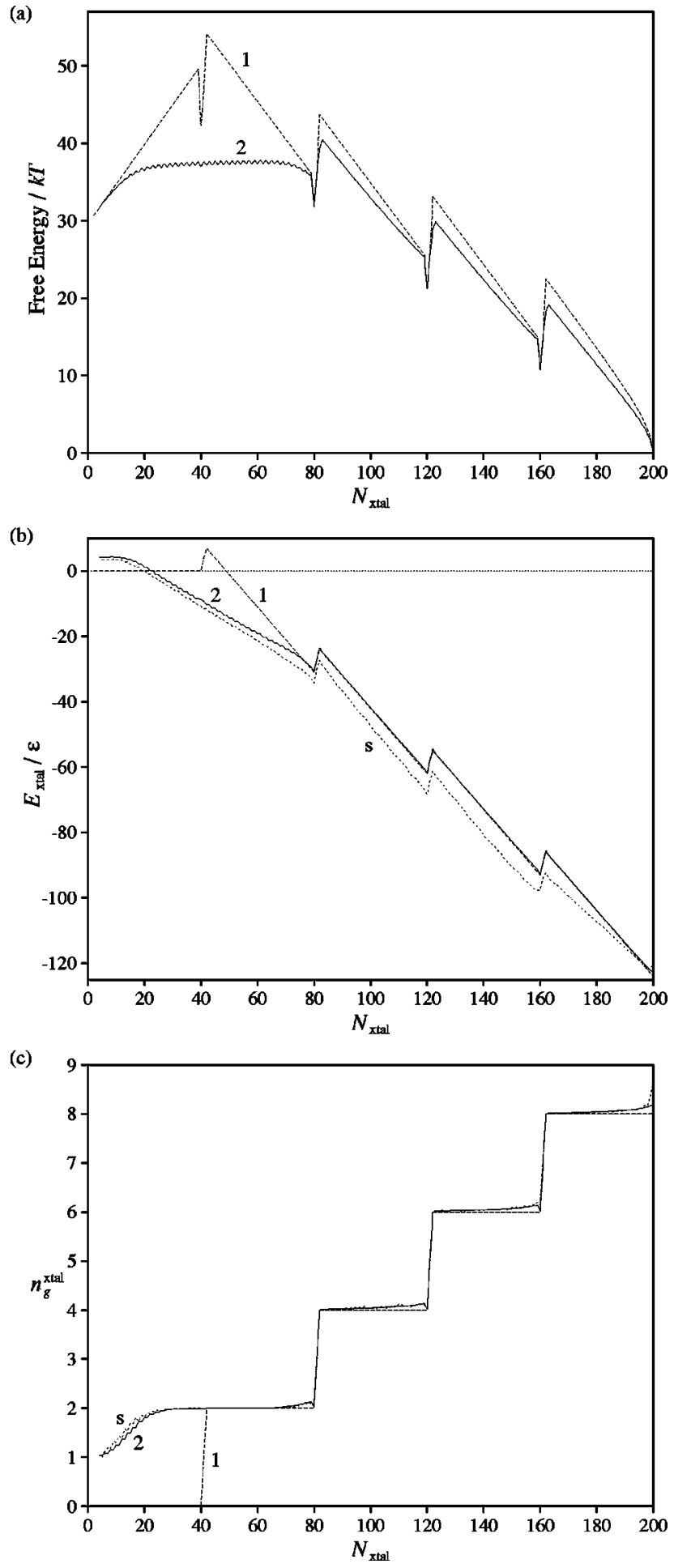

FIG. 8. Comparison of results calculated using Eq. (9) for crystallization pathways which allow one (the dashed line 1) and two (the solid line 2) incomplete stems to simulation (the dotted line s) results. (a) Free energy for the formation of the crystal, (b) energy of the crystal (excluding the contribution from interactions between the polymer and the surface), $E_{x \text { tal }}$, and (c) number of gauche bonds in the crystal, $n_{g}^{x \text { tal }}$, as a function of the number of units in the crystal. The crystal has stems 40 units long. $T=2.05 \epsilon k^{-1}$.

nucleation barrier occurs near $N_{\text {stem }}=1 \quad\left(N_{x \text { tal }}=l\right)$ and is a much steeper function of $l$.

To gain some further insight into the free energy profiles obtained from the simulation, we attempt to model them us- 
ing a simple calculation in which we only explicitly consider the crystalline part of the polymer configuration and assume the rest behaves like an ideal two-dimensional coil. The free energy is then

$$
A\left(N_{\text {xtal }}\right)=A_{\text {coil }}\left(N_{\text {rest }}\right)+\sum \exp \left(-\beta\left(E_{\text {xtal }}+E_{\text {join }}\right)\right),
$$

where the sum is over all possible configurations for the crystalline portion of the chain which are $N_{x t a l}$ units long, $E_{x t \text { tal }}$, the energy of the crystal, is given by Eq. (1), $A_{\text {coil }}$, the free energy of the ideal two-dimensional coil, is given by

$$
A_{\text {coil }}\left(N_{\text {rest }}\right)=-N_{\text {rest }} k T \log \left(1+2 \exp \left(-\beta \epsilon_{g}\right)\right)-N_{\text {rest }} \epsilon,
$$

and $E_{\text {join }}$ is the energy of any gauche bonds that might by necessity occur at the junction between the crystalline and the coil parts of the chain. (The end of an incomplete stem in the crystalline portion of the chain must either occur at an end of the chain or be followed by a gauche bond.)

Some free energy profiles obtained from this approach are shown in Fig. 7(c). They have a remarkably similar structure to the simulation results, although there are two main discrepancies. First, our expression for $A_{\text {coil }}$ is an underestimate of the true free energy because it neglects the energetic contribution from contacts between different parts of the coil. Therefore, Eq. (9) overestimates the stability of the crystal compared to simulation results at the same temperature. Second, we do not take into account the fact that structural patterns corresponding to small crystal nuclei naturally occur in the disordered state, and so the calculated profiles do not have a large rise in free energy at small values of $N_{x \text { tal }}$.

The useful feature of this simple calculation is that it allows us to understand some of the physical origins of the features in the free energy profile more easily. For example, $A\left(N_{x \text { tal }}=n l\right)$ is always significantly lower than $A\left(N_{x \text { tal }}\right.$ $=n l-1)(n \geqslant 2)$; this sharp dip in the free energy is because for $N_{x \mathrm{tal}}=n l$ neither end of the crystalline portion has to be terminated by a gauche bond, i.e., $E_{\text {join }}=0$.

More interestingly, it can give greater insight into the effects of having a two-stem nucleus. In Fig. 8 we compare the results when all possible crystalline configurations are considered in the sum of Eq. (9) and when only those in which there is a single incomplete stem are considered. In the latter case the initial nucleus must be a single stem, and so the results should be much closer to the surface nucleation profile. They are; the relevant free energy profile in Fig. 8(a) now shows a free energy barrier for the formation of the first fold at $N_{x t a l}=l$ and there is a steep initial rise in the free energy. However, it can be clearly seen that the free energy is much lower when the possibility of a two-stem nucleus is allowed; this is because the energy of a sufficiently long two-stem nucleus is lower than a single stem [Fig. 8(b)]. Furthermore, when assuming a single-stem nucleus the results for $E_{x \text { tal }}$ and $n_{g}^{x \text { tal }}[$ Fig. 8(c)], as well as the free energy, significantly differ from simulation results, whereas the calculation and simulation are in good agreement when the twostem nucleus is allowed. (The slightly lower value of $E_{x \text { tal }}$ for the simulation results is due to contacts between the crystalline portion and the rest of the chain. These are not taken into account in our simple calculation.)

The calculated profiles have two slopes for $N_{x t a l}<2 l$. For small values of $N_{x \text { tal }}$ the profile is steeper (and always positive) because it is still more favorable to have a singlestem nucleus. (This feature has no parallel in the simulation profile; instead, as discussed earlier there is a rapid rise in free energy as $N_{x t a l}$ becomes smaller.) In this range the two calculated profiles in Fig. 8(a) are very similar. In the range $N_{x t a l}=15-20$ the profile changes slope as the structure of the nucleus changes. Assuming an ideal two-stem nucleus the slope of the free energy profile for $20 \leqq N_{x t a l}<2 l$ will be

$$
\frac{d A}{d N_{x \mathrm{tal}}}=-\frac{\epsilon}{2}+k T \log \left(1+2 \exp \left(-\beta \epsilon_{g}\right) .\right.
$$

Therefore, the profile in this region is expected to be flat for $T=2.03 \epsilon k^{-1}$ [Fig. 8(a)] and below this temperature the calculated profile will exhibit a maximum at small values of $N_{x t a l}$ [e.g., $T=1.7 \epsilon k^{-1}$ Fig. 7(c)]. A similar temperature dependence of the slope for $N_{x \mathrm{tal}} \sim 20-80$ is seen in the simulation free energy profiles [Fig. 7(a)].

\section{v. CONCLUSIONS}

The thermodynamic properties that we find for our semiflexible polymer in the presence of a surface have many similarities to an isolated semi-flexible polymer, ${ }^{22,25,26,28}$ e.g., the coexistence of chain-folded crystallites with different aspect ratio and the order-disorder transition. (It should be remembered that the formation of folded structures for our single polymer system is a thermodynamic effect, whereas lamellar polymer crystals have folded chains due for kinetic reasons.) One new feature is the desorption transition ${ }^{14}$ in which the adsorbed two-dimensional polymer gradually adopts a three-dimensional configuration. Also of interest is the fact that crystallization is always preceded by adsorption. However, it is not yet clear whether this feature is likely to be common to many polymers or reflects some of the aspects of our current model.

The free energy profiles that we obtain by umbrella sampling for specific crystallization pathways have a sawtooth structure where each rise in free energy corresponds to the formation of a new fold. We found that the apportionment factor, $\Psi\left(N_{\text {stem }} \rightarrow N_{\text {stem }}+1\right) \approx 0$ for $N_{\text {stem }} \geqslant 2\left(N_{\text {stem }}\right.$ is the number of stems in the crystalline configuration). However for $N_{\text {stem }}<2$ our free energy profiles are significantly different from those of Lauritzen and Hoffman's surface nucleation theory. There is no feature in our profiles that corresponds to the formation of the first fold because the initial nucleus is not a single stem but two incomplete stems connected by a single fold. The nucleus increases in size by the simultaneous growth of both stems. The reason that a twostem nucleus is preferred is simply because beyond a certain size it has a lower energy than a single-stem nucleus. Such energetic considerations are also likely to hold for more realistic polymer models. This finding has serious implications for the coherence of the surface nucleation theory of polymer 
crystallization because the barrier to the formation of the first stem, and its dependence on crystal thickness, plays a key role in the theory.

Our results also hint at another potential problem with the surface nucleation theory. To obtain the free energy profiles of Fig. 7 we had to constrain the system to prevent crystallization occurring by other pathways. In surface nucleation theory only a small subset (those where the stem length is constant) of the multitude of possible pathways are taken into account. However, a rigorous treatment requires that the full web of possible paths is explored. ${ }^{48-50}$ Recent kinetic Monte Carlo simulations which do just this present a radically different picture of the mechanism by which the thickness of lamellar crystals is determined. ${ }^{30,31}$

\section{ACKNOWLEDGMENTS}

The work of the FOM Institute is part of the research program of "Stichting Fundamenteel Onderzoek der Materie" (FOM) and is supported by NWO ("Nederlandse Organisatie voor Wetenschappelijk Onderzoek"). J.P.K.D. acknowledges the financial support provided by the Computational Materials Science program of the NWO. We thank James Polson and Wim de Jeu for a critical reading of the manuscript.

${ }^{1}$ A. Keller, Philos. Mag. 2, 1171 (1957).

${ }^{2}$ For a balanced theoretical review see K. Armistead and G. GoldbeckWood, Adv. Polym. Sci. 19, 219 (1992).

${ }^{3}$ J. I. Lauritzen and J. D. Hoffman, J. Res. Natl. Bur. Stand., Sect. A 64, 73 (1960).

${ }^{4}$ J. D. Hoffman, G. T. Davis, and J. I. Lauritzen, in Treatise on Solid State Chemistry, edited by N. B. Hannay (Plenum, New York, 1976), Vol. 3, Chap. 7, p. 497.

${ }^{5}$ J. D. Hoffman and R. L. Miller, Polymer 38, 3151 (1997)

${ }^{6}$ D. M. Sadler and G. H. Gilmer, Polymer 25, 1446 (1984).

${ }^{7}$ D. M. Sadler and G. H. Gilmer, Phys. Rev. Lett. 56, 2708 (1986).

${ }^{8}$ D. M. Sadler and G. H. Gilmer, Phys. Rev. B 38, 5684 (1988).

${ }^{9}$ M. A. Spinner, R. W. Watkins, and G. Goldbeck-Wood, J. Chem. Soc., Faraday Trans. 91, 2587 (1995).

${ }^{10}$ T. Yamamoto, J. Chem. Phys. 107, 2653 (1997).

${ }^{11}$ C.-M. Chen and P. G. Higgs, J. Chem. Phys. 108, 4305 (1998).
${ }^{12}$ L. Toma, S. Toma, and J. A. Subirana, Macromolecules 31, 2328 (1998).

${ }^{13}$ C. Liu and M. Muthukumar, J. Chem. Phys. 109, 2536 (1998).

${ }^{14}$ E. Eisenriegler, Polymers Near Surfaces (World Scientific, Singapore, 1993).

${ }^{15}$ E. Eisenriegler, K. Kremer, and K. Binder, J. Chem. Phys. 77, 6296 (1982).

${ }^{16}$ H. Meirovitch and S. Livne, J. Chem. Phys. 88, 4507 (1988).

${ }^{17}$ I. Chang and H. Meirovitch, Phys. Rev. Lett. 69, 2232 (1992).

${ }^{18}$ D. P. Foster, E. Orlandi, and M. C. Tesi, J. Phys. A 25, L1211 (1992).

${ }^{19}$ K. De'Bell and T. Lookman, Rev. Mod. Phys. 65, 87 (1993).

${ }^{20}$ P.-Y. Lai, Phys. Rev. E 49, 5420 (1994).

${ }^{21}$ A. Milchev and K. Binder, Macromolecules 29, 343 (1996).

${ }^{22}$ A. Kolinski, J. Skolnick, and R. Yaris, J. Chem. Phys. 85, 3585 (1986).

${ }^{23}$ Y. A. Kuznetsov, E. G. Timoshenko, and K. A. Dawson, J. Chem. Phys. 104, 336 (1996).

${ }^{24}$ Y. Zhou, C. K. Hall, and M. Karplus, Phys. Rev. Lett. 77, 2822 (1996).

${ }^{25}$ S. Doniach, T. Garel, and H. Orland, J. Chem. Phys. 105, 1601 (1996).

${ }^{26}$ U. Bastolla and P. Grassberger, J. Stat. Phys. 89, 1061 (1997).

${ }^{27}$ S. Fujiwara and T. Sato, J. Chem. Phys. 107, 613 (1997).

${ }^{28}$ J. P. K. Doye, R. P. Sear, and D. Frenkel, J. Chem. Phys. 108, 2134 (1998).

${ }^{29}$ H. Noguchi and K. Yoshikawa, Chem. Phys. Lett. 278, 184 (1997).

${ }^{30}$ J. P. K. Doye and D. Frenkel, Phys. Rev. Lett. 81, 2160 (1998).

${ }^{31}$ J. P. K. Doye and D. Frenkel, J. Chem. Phys. (submitted) (cond-mat/ 9807357).

${ }^{32}$ J. I. Siepmann and D. Frenkel, Mol. Phys. 75, 59 (1992).

${ }^{33}$ M. Dijkstra, D. Frenkel, and J. P. Hansen, J. Chem. Phys. 101, 3179 (1994).

${ }^{34}$ R. Ramakrishnan, B. Ramachandran, and J. F. Pekny, J. Chem. Phys. 106, 2418 (1997).

${ }^{35}$ J. M. Deutsch, J. Chem. Phys. 106, 8849 (1997).

${ }^{36}$ A. M. Ferrenberg and R. H. Swendsen, Phys. Rev. Lett. 63, 1195 (1989).

${ }^{37}$ R. Poteau, F. Spiegelman, and P. Labastie, Z. Phys. D 30, 57 (1994).

${ }^{38}$ G. M. Torrie and J. P. Valleau, J. Comput. Phys. 23, 187 (1977).

${ }^{39}$ B. A. Berg and T. Neuhaus, Phys. Lett. B 267, 249 (1991).

${ }^{40}$ B. A. Berg and T. Neuhaus, Phys. Rev. Lett. 69, 9 (1992).

${ }^{41}$ B. A. Berg, J. Stat. Phys. 82, 323 (1996).

${ }^{42} \mathrm{G}$. Ungar et al., Science 229, 386 (1985).

${ }^{43}$ S. J. Organ, A. Keller, M. Hikosaka, and G. Ungar, Polymer 37, 2517 (1996).

${ }^{44}$ H. S. Chan et al., J. Chem. Phys. 94, 8542 (1991).

${ }^{45}$ C. R. Snyder, H. Marand, and M. L. Mansfield, Macromolecules 29, 7508 (1995).

${ }^{46}$ C. R. Snyder and H. Marand, Macromolecules 30, 2759 (1997).

${ }^{47}$ J.-J. Point, Faraday Discuss. Chem. Soc. 68, 167 (1979).

${ }^{48}$ J. J. Point, Macromolecules 12, 770 (1979).

${ }^{49}$ J.-J. Point, Faraday Discuss. Chem. Soc. 68, 366 (1979).

${ }^{50}$ E. A. DiMarzio and C. M. Guttman, J. Appl. Phys. 53, 6581 (1982). 